\title{
Ruptured Pancreaticoduodenal Artery Aneurysm Associated with Median Arcuate Ligament Compression and Aortic Dissection Successfully Treated with Embolotherapy
}

\author{
Shiro Miyayama, MD, ${ }^{1}$ Takuro Terada, MD, ${ }^{2}$ and Masato Tamaki, MD ${ }^{3}$
}

A 51-year-old man with a ruptured pancreaticoduodenal artery (PDA) aneurysm caused by compression of the celiac artery by the median arcuate ligament and aortic dissection involving the celiac axis was transferred to our hospital for endovascular treatment. A 4-F catheter was advanced into the superior mesenteric artery through the narrow true lumen via the left brachial artery, and coil embolization of the aneurysm was successfully performed. In this case, rapid increase of blood flow in the superior mesenteric artery, which compensated for the decreased celiac blood flow by aortic dissection, increased hemodynamic stress on the PDA, leading to aneurysmal rupture.

Keywords: pancreaticoduodenal artery aneurysm, median arcuate ligament, aortic dissection

\section{Introduction}

Celiac artery stenosis due to compression by the median arcuate ligament (MAL) causes ruptured pancreaticoduodenal artery (PDA) aneurysm. ${ }^{1-4)}$ In addition, a sudden hemodynamic change in the PDA, such as localized celiac artery dissection, ${ }^{5)}$ also causes PDA aneurysm rupture.

We encountered a patient with a ruptured PDA aneurysm associated with MAL compression and aortic dissection involving the celiac trunk, who successfully underwent endovascular treatment. In this report, we describe the technical aspects of our case.

${ }^{1}$ Department of Diagnostic Radiology, Fukuiken Saiseikai Hospital, Fukui, Fukui, Japan

${ }^{2}$ Department of Surgery, Fukuiken Saiseikai Hospital, Fukui, Fukui, Japan

${ }^{3}$ Department of Surgery, Fukui Cardiovascular Center, Fukui, Fukui, Japan

Received: September 30, 2014; Accepted: December 1, 2014 Corresponding author: Shiro Miyayama, MD. Department of

Diagnostic Radiology, Fukuiken Saiseikai Hospital, 7-1,

Funabashi, Wadanaka-cho, Fukui, Fukui 918-8503, Japan

Tel: +81-776-23-1111, Fax: +81-776-28-8519

E-mail: s-miyayama@fukui.saiseikai.or.jp

\section{Case Report}

A 51-year-old man suddenly presented with abdominal pain and was admitted to another hospital. Computed tomography (CT) showed a massive retroperitoneal hematoma and Stanford type B aortic dissection that involved the celiac axis and bilateral iliac arteries (Fig. 1). Celiac artery stenosis due to MAL was also demonstrated. Emergent laparotomy was performed because rupture of the aorta or its branches was suspected. However, active bleeding was not identified during the laparotomy and the cause of retroperitoneal hematoma was uncertain. The operation was discontinued without surgical intervention. Follow-up CT 1 week after the operation demonstrated a PDA aneurysm, which showed gradual enlargement on CT at 14 and 18 days after the operation, respectively (Fig. 2A and B). The patient was transferred to our hospital for endovascular treatment of the PDA aneurysm 19 days after the operation.

The left brachial artery was punctured and a 4-F sheath (Terumo, Tokyo, Japan) was inserted because the dissection involved the bilateral iliac arteries and selective catheterization into the superior mesenteric artery seemed to be difficult via the femoral access through the narrow true lumen (Fig. 2C). After confirmation of the true lumen by left anterior oblique aortography (Fig. 3A), a J-shaped 4-F catheter (Terumo, Japan) was advanced into the superior mesenteric artery via the true lumen. Superior mesenteric arteriography showed a PDA aneurysm and opacification of the celiac arterial branches through the PDA (Fig. 3B). A 2-F tip microcatheter (Excelsior, Boston Scientific, Natick, Massachusetts, USA) was advanced distal to the PDA aneurysm using a 0.016-inch guidewire (ASAHI Meister, Asahi Intecc, Seto, Japan). Metallic coils (IDC, Boston Scientific, USA) were deployed from the distal to proximal portions of the aneurysm. Superior mesenteric arteriography immediately after coil embolization showed the disappearance of the PDA aneurysm and opacification of the celiac arterial branches via the PDAs (Fig. 3C). The patient was discharged from 


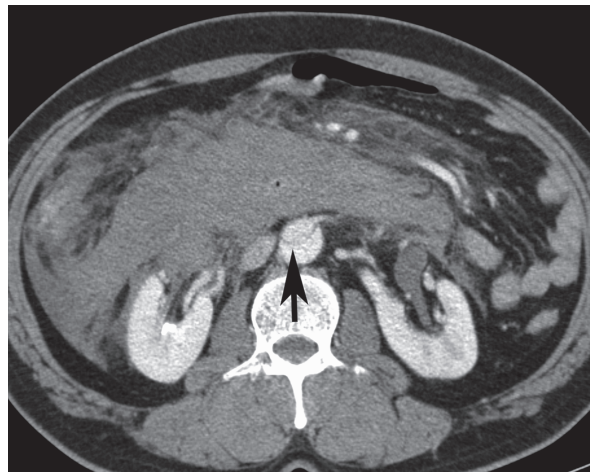

Fig. 1 Contrast-enhanced computed tomography (CT) obtained immediately after admission to another hospital showed a massive retroperitoneal hematoma and aortic dissection (arrow).

our hospital 1 week after coil embolization. Aortic dissection was managed conservatively at the previous hospital for 2 years and 9 months, without any symptoms.

\section{Discussion}

PDA aneurysms account for $2 \%$ of all visceral artery aneurysms, ${ }^{6}$ and are often accompanied by pancreatitis, trauma including surgery, and occlusion or stenosis of the celiac artery due to arteriosclerosis or MAL compression. ${ }^{1-6)}$ Segmental arterial mediolysis also causes PDA aneurysms.7) The incidence of PDA aneurysm rupture is up to $65 \%, 1,3,6,8)$ and the risk of rupture appears to be unrelated to the size of the aneurysm. ${ }^{4}$ In a review of 88 patients with PDA aneurysms, 53 experienced rupture, which was fatal in $\left.26(49.1 \%){ }^{8}\right)$

MAL is a fibrous arch that connects the left and right diaphragmatic cruras, defining the anterior margin of the aortic hiatus, and causes celiac artery stenosis by extrinsic compression. ${ }^{9)}$ Multi-planer reconstruction CT and 3-dimensional CT angiography can clearly demonstrate the compression of the celiac axis, with a characteristic hooked appearance caused by MAL.9) Repeated compression of the celiac artery by MAL may also have resulted in traumatic changes that cause dissection. ${ }^{5)}$

We speculate that our patient had already had an increased blood flow related to celiac stenosis caused by MAL. In addition to such a background, aortic dissection that involved the celiac trunk suddenly caused a further decrease in the blood flow in the celiac artery. The decreased blood flow in the celiac artery was compensated for the increased blood flow through the PDA, and this rapid increase in the blood flow elevated hemodynamic stress on the PDA, promoting bleeding. However, it was uncertain whether this patient had already had a true PDA
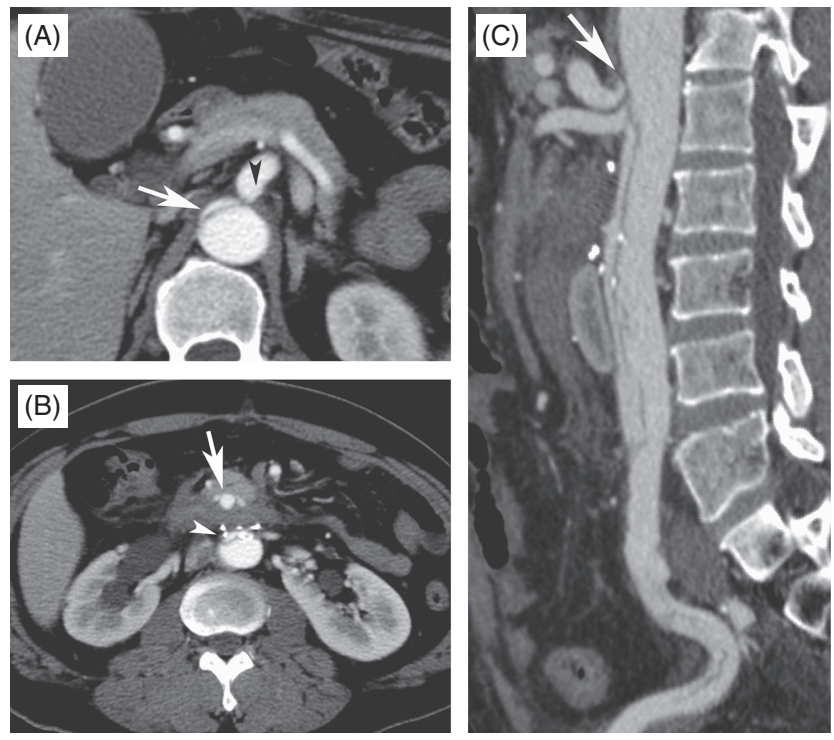

Fig. 2 Computed tomography (CT) obtained 18 days after the operation. (A) Arterial phase CT showed aortic dissection involving the celiac axis (arrowhead). The arrow indicates the narrow true lumen. (B) A pancreaticoduodenal artery (PDA) aneurysm was also demonstrated (arrow) at a lower level. The arrowhead indicates the true lumen. (C) Reconstructed curved sagittal CT showed the characteristic hooked appearance of the celiac axis caused by median arcuate ligament (MAL) compression (arrow). The dissection was reached the right external iliac artery.

aneurysm before the onset of aortic dissection because the aneurysm was thought as a pseudoaneurysm. To the best of our knowledge, only one Japanese patient of ruptured PDA aneurysm associated with aortic dissection involving the celiac axis has been reported. ${ }^{10)}$

Two strategies are available for the management of a PDA aneurysm: endovascular treatment and surgical resection. ${ }^{1-8)}$ Endovascular treatment, which includes coil embolization and stent grafting, is less invasive than surgical resection. Therefore, endovascular treatment should be attempted first, if possible. Catheter-guidewire manipulation in cases with subacute phase dissecting lesions may carry the risk of aortic rupture. ${ }^{7)}$ However, recent advanced catheter-guidewire technologies allow safe endovascular treatment even in such cases. We chose the left brachial artery approach because catheter insertion into the true lumen seemed straightforward. Selective catheterization into the superior mesenteric artery could also be easily performed through the narrow true lumen. We consider that endovascular treatment of an aortic branch via a dissected lumen is not always contraindicated when a safe approach route can be selected.

Treatment of celiac artery stenosis in addition to the aneurysm therapy remains controversial. Relieving the MAL compression would theoretically decrease recurrence 

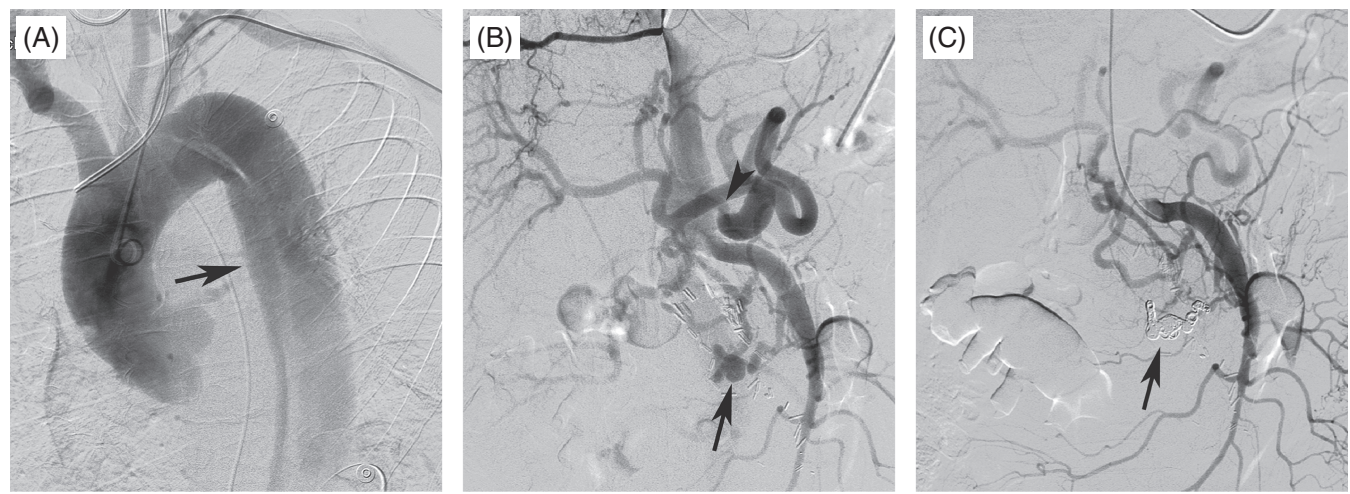

Fig. 3 Arteriogram obtained during the interventional procedure. (A) Left anterior oblique thoracic aortography showed aortic dissection. The arrow indicates the narrow true lumen. The catheter was advanced into the true lumen. (B) Right anterior oblique abdominal aortography via the true lumen showed a pancreaticoduodenal artery (PDA) aneurysm (arrow) and median arcuate ligament (MAL) compression (arrowhead). Bilateral renal arteries were not opacified because they arose from the false lumen. (C) Right anterior oblique superior mesenteric arteriography immediately after coil embolization showed the disappearance of the PDA aneurysm and opacification of the celiac branches through the PDAs. The arrow indicates the metallic coils.

of aneurysms in the PDA, although no recurrences have been reported in the literature after successful embolization of the aneurysm alone. ${ }^{3,4)}$ In this case, long-term follow-up is required to check the recurrence of aneurysms because the hemodynamic stress on the PDA still remains after the treatment.

In summary, we report a case of a ruptured PDA aneurysm caused by MAL and aortic dissection. Endovascular treatment could be safely performed through a narrow true lumen.

\section{Disclosure Statement}

The authors have no conflict of interest to declare.

\section{References}

1) Quandalle P, Chambon JP, Marache P, et al. Pancreaticoduodenal artery aneurysms associated with celiac axis stenosis: report of two cases and review of the literature. Ann Vasc Surg 1990; 4: 540-5.

2) Suzuki K, Kashimura H, Sato M, et al. Pancreaticoduodenal artery aneurysms associated with celiac axis stenosis due to compression by median arcuate ligament and celiac plexus. J Gastroenterol 1998; 33: 434-8.

3) Kallamadi R, Demoya MA, Kalva SP. Inferior pancreaticoduodenal artery aneurysms in association with celiac stenosis/occlusion. Semin Intervent Radiol 2009; 26: 215-23.

4) Takase A, Akuzawa N, Hatori T, et al. Two patients with ruptured posterior inferior pancreaticoduodenal artery aneurysms associated with compression of the celiac axis by the median arcuate ligament. Ann Vasc Dis 2014; 7: 87-92.

5) Watanabe A, Kohtake H, Furui S, et al. Celiac artery dissection seen with ruptured pancreaticoduodenal arcade aneurysms in two cases of celiac artery stenosis from compression by median arcuate ligament. J Vasc Surg 2012; 56: 1114-8.

6) Shanley CJ, Shah NL, Messina LM. Uncommon splanchnic artery aneurysms: pancreaticoduodenal, gastroduodenal, superior mesenteric, inferior mesenteric, and colic. Ann Vasc Surg 1996; 10: 506-15.

7) Ushijima T, Izumo A, Matsumoto T, et al. Pancreaticoduodenal artery pseudoaneurysm caused by segmental arterial mediolysis: a case report of surgical treatment. Ann Vasc Dis 2013; 6: 198-201.

8) de Perrot M, Berney T, Deléaval J, et al. Management of true aneurysms of the pancreaticoduodenal arteries. Ann Surg 1999; 229: 416-20.

9) Horton KM, Talamini MA, Fishman EK. Median arcuate ligament syndrome: evaluation with CT angiography. Radiographics 2005; 25: 1177-82.

10) Sakatani A, Kitayama S, Miyabe H, et al. Ruptured pancreaticoduodenal artery aneurysm due to celiac axis occlusion caused by aortic dissection. Nihon Fukubu Kyukyu Igakkai Zasshi. 2011; 31 (suppl) 275 (in Japanese). 